CLAWAR2016: 19th International Conference on Climbing and Walking Robots and the Support Technologies for

Mobile Machines, London, UK, 12-14 September 2016, World Scientific Publishing Company, Singapore, ISBN:

978-981-3149-12-0.

\title{
PD-FUZZY CONTROL OF LOWER LIMB EXOSKELETON FOR ELDERLY MOBILITY
}

\author{
GHASAQ ALREZAGE, ABDULLAH ALSHATTI and M. O. TOKHI \\ Department of Automatic Control and System Engineering, \\ University of Sheffield, UK \\ Gsmousa1@sheffield.ac.uk
}

\begin{abstract}
This paper presents investigations into the development of a control strategy lower limb exoskeleton device for elderly people. The exoskeleton is designed to enhance the lower limb capability and provide support torque to augment the torque of knee and hip during the walking cycle. Proportional derivative fuzzy logic controls are designed and implemented in this work. The visual Nastran 4D software is used to develop a simulation model of the exoskeleton and a humanoid for test and verification of the developed control mechanism. Simulation results demonstrating the performance of the adopted approach are presented and discussed.
\end{abstract}

\section{INTRODUCTION}

The increasing trend in ageing population has become a challenge on a global scale. The World Health Organization (WHO) statistics show that the birth rate in all regions has decreased, and the proportion of old people above 60 has increased from $8 \%$ in 1950 to $10 \%$ in 2000 and is estimated to rise to $21 \%$ by 2050 [1]. Walking is one of the most common human features. Normally, the elderly people cannot keep their step length and their leg muscle power; most people will experience mobility problems when they become old. This situation is becoming more serious, especially, in developed countries. Many types of transport mechanism such as the classic crutch or wheelchair have been developed to help the elderly. These are of limited benefit.

A key development in addressing the problem of mobility could be the exoskeleton system, which is the focus of this paper. The paper is organized as follows: Section 2 presents a brief background of the exoskeleton, Sections 3 and 4 describe the humanoid model and the designed exoskeleton with the control structure, and Section 5 presents the simulation results and the paper is concluded in Section 6. 


\section{EXOSKELETONS - A BRIEF BACKGROUND}

The assistive exoskeleton is considered as one of the most salutary inventions of modern science [2]. It is a type of incorporation mechanism, which can be worn by elderly to enhance and augment their lower limb strength [3]. Significant number of researchers have considered the development of assistive devices for empowering or rehabilitation. The aim is to augment reduced power of the joint and carry part of the body weight or use as aid and substitute the human limb [4, 5].

Exoskeletons used for rehabilitation could be classified into two types: the immobile robot exoskeleton and the over ground exoskeleton. Earlier exoskeletons used for rehabilitation were treadmill based gait devices. This type of system was originally designed to enable the patient to have gait training in a rigid and confined space $[6,7]$. A system for body weight support was used in the treadmill, predetermined pattern for walking was used, and normally variation was not allowed within the gait pattern. Sometimes, virtual reality environment was used, that may both induce and power the patient to perform the movement actively.

The roboknee was produced in 2004 to enhance and strengthen the wearer's muscle. The system uses a series elastic actuator to produce low impedance and high force. PD controller is used to control the force. Therefore, the main purpose of the roboknee is to enhance the torque of the human joints $[8,9]$. In 2005 the University of North-eastern developed an active knee rehabilitation device (AKROD). The goal of the device was to help stroke patients by providing rehabilitation and improving the gait restoration process. They used electro rheological fluid actuator on one side to create a resistance to the movement of the healing joint to help the muscle strength [10].

Hybrid assistive limb (HAL), as a wearable robot has been designed by the Japanese company, Cyberdyne for different functions. The main goal is to help elderly people in their walking. HAL consists of three main parts: skeleton and actuator, controller and sensor. Aluminum alloy and steel are used in the construction. DC actuator used for generating the required torque for both hip and knee, measuring unit, power supply and the controller are included in the backpack. Force sensor is used in the sole of the foot to measure ground reaction force, while EMG measurement sensor is attached to skin surface of leg to estimate the muscle activity [11].

Wearable walking helper (WWH) is a gravity compensation wearable exoskeleton developed to enhance the ambulate functions of disabled and elderly people [12]. The torque provided by WWH to assist the locomotion of 
patient is proportional to the torque calculated based on approximated human model. Another exoskeleton developed to provide assistance to the elderly people in walking is walking power assist leg (WPAL) [13]. It uses a hypird control mechanism based on joint angles, velocities and interaction forces.

\section{SYSTEM MODEL}

\subsection{Humanoid}

The humanoid model is developed so as to resemble human-like physical and functional characteristics [14]. The human model segment parameters were built according to the anthropometric data presented by Winter (2009). A humanoid model of $75 \mathrm{~kg}$ weight and $1.7 \mathrm{~m}$ height is used in this work. The humanoid was designed in Visual Nastran 4D (VN4D) software environment. The VN4D allows development of simulation models of complex mechanical systems easily [15]. Furthermore, it links with Matlab/Simulink for development and testing of controllers. In the humanoid model the hip and knee joints follow reference trajectories from Clinical Gate Analysis (CGA) data, which was collected by recording video motion for standard walking cycle. While the ankle motion is defined as:

$$
\begin{aligned}
& X \text { ankle }=\mathrm{L}_{\text {th }} \sin \left(\boldsymbol{\theta}_{\boldsymbol{h}}\right)+\mathrm{L}_{\text {sh }} \sin \left(\boldsymbol{\theta}_{\boldsymbol{k}}\right) \\
& Y \text { ankle }=\mathrm{L}_{\text {th }} \cos \left(\boldsymbol{\theta}_{\boldsymbol{h}}\right)+\mathrm{L}_{\text {sh }} \cos \left(\boldsymbol{\theta}_{\boldsymbol{k}}\right)
\end{aligned}
$$

where $\boldsymbol{\theta}_{\boldsymbol{h}}$, and $\boldsymbol{\theta}_{\boldsymbol{k}}$ are the hip and knee joint angles respectively. $\mathrm{L}_{\mathrm{th}}$, and $\mathrm{L}_{\mathrm{sh}}$ represent the length or height of thigh, and shank respectively.

These where $\mathrm{L}_{\mathrm{th}}=416.5 \mathrm{~mm}, \mathrm{~L}_{\mathrm{sh}}=418.2 \mathrm{~mm}$ in this work.

\subsection{Exoskeleton}

The exoskeleton can perform many functions, and its main purpose in this work is to assist mobility for elderly. The second purpose is to provide assistive torque to reduce the torque (effort required) of the humanoid joint during the walking cycle. Light weight and low cost material are the main mechanical design considerations. Glass fiber reinforced polymer (GFRP) is used in this work. The exoskeleton was designed using solid works software. It provides back support and consists of waist, thigh, and shank parts, and soles, hip and knee joints [16]. Sensors are used to provide feedback information based on measurements of orientation and torque at hip, knee and ankle joints. The final design was incorporated into the humanoid model as shown in Figure 1. 


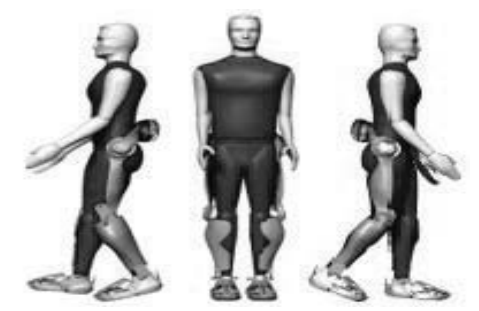

Figure 1. Humanoid and exoskeleton joint.

\section{CONTROL STRUCURE}

The control methodology plays significant part in controlling the exoskeleton. The exoskeleton is required to follow the human movement and this makes the exoskeleton system more complex [1, 17]. It is evident from the review of previous work that the main issue of elderly mobility is body weight. Thus, support should be provided by the exoskeleton system to the user. The fundamental goal of the control method is to measure the force required at each lower limb joints during the walking cycle, then the exoskeleton system should be controlled in a way to provide an assistive force to the user. Two separate controllers are devised, one for the humanoid model and one for the exoskeleton providing $60 \%$ and $40 \%$ of the overall torque respectively, i.e. $60 \%$ of power will drive the humanoid, and the other $40 \%$ of the power will drive the exoskeleton. The orientation will be controlled for the humanoid lower limb to follow predefined reference trajectory. The torque of each joint is calculated during the walking cycle.

\subsection{Fuzzy Logic Controller}

Fuzzy logic control (FLC) is considered in this work for control of the humanoid and the exoskeleton. Mamdani type fuzzy rule is used with multi-input single output (MISO) structure as shown in Figure 2. The inputs of the system are the error and change of error. The orientation of lower limb joints (hip and knee) are independently controlled by PD-fuzzy logic structures which the similar rule bases. Thus, there are four independent FLCs (FLC1, FLC2, FLC3, and FLC4), one for each joint integrating two inputs and one output for each FLC. Five Gaussian membership functions (MFs) are used. These are positive big (PB), positive small (PS), zero (Z), negative small (NS), and negative big (NB). All FLCs use orientation error, e, and change orientation error, $\Delta$ e. The same design is used for all FLCs, thus, only one fuzzy logic controller structure is implemented for all the situations. The fuzzy rule base is shown in Table 1. 


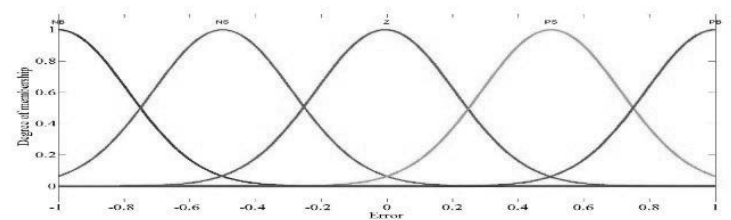

Figure 2. Input output and MFs of MISO system.

Table 1 . The fuzzy rule.

\begin{tabular}{|c|c|c|c|c|c|}
\hline$e \Delta e$ & NS & NB & $z$ & PS & PB \\
\hline NS & NB & NB & NS & NS & $z$ \\
\hline NB & NB & NS & NS & $z$ & PS \\
PS & NS & NS & $z$ & PS & PS \\
\hline PB & NS & $Z$ & PS & PS & PB \\
\hline
\end{tabular}

Four PD-like fuzzy logic controllers (FLCs) are developed in this work. The PD-fuzzy control is designed in Matlab Simulink linked with VN environment. The control structure is shown in Figure 3. PD gains and the fuzzy scaling factors were tuned heuristically.

The objective of the proposed control approach is to provide an assistive torque to enhance the capability of elderly people to walk. The GCA data is used as reference in this work. The outputs of the controller are the torque of left hip, left knee ( $\tau l k)$, right hip ( $\tau r h)$, and right knee ( $\tau r k)$. These outputs are used to control the hip and knee joints based on the corresponding references, which are sent to both the humanoid and the exoskeleton actuators in VN4D.

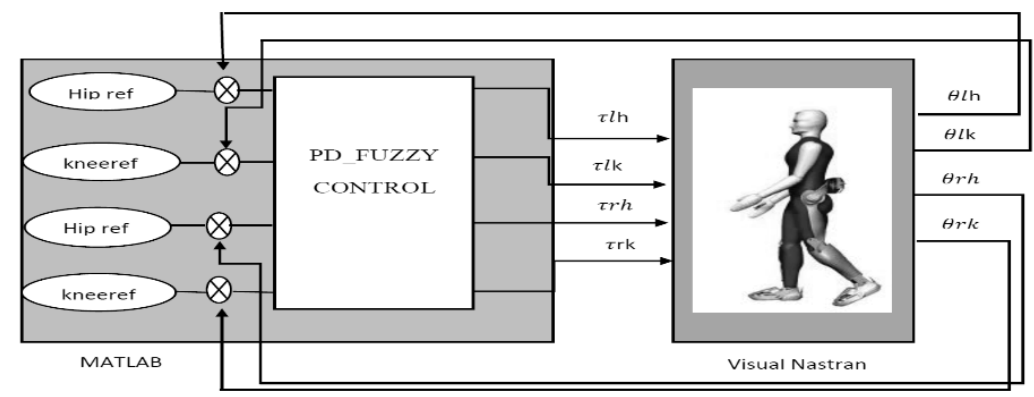

Figure 3. Control system structure.

\section{SIMULATION RESULTS}

Reference tracking performances of the control system for hip and knee joints are shown in Figures 4 and 5. The red solid line is the actual trajectory, and blue 
line is the desired trajectory. It is noted that the PD fuzzy logic controller has achieved good tracking performance than the conventional PID a previous work of the authors [16].
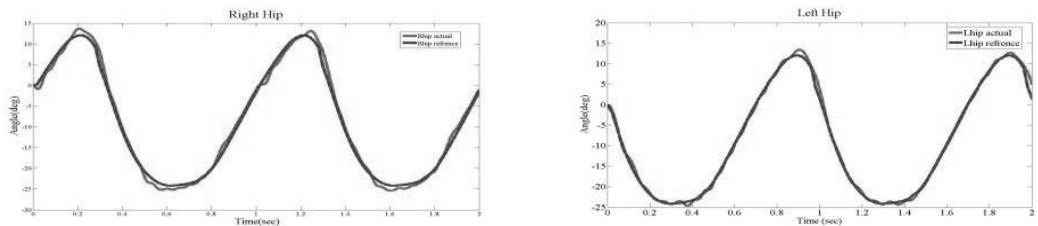

Figure 4. Hip joint trajectory tracking.
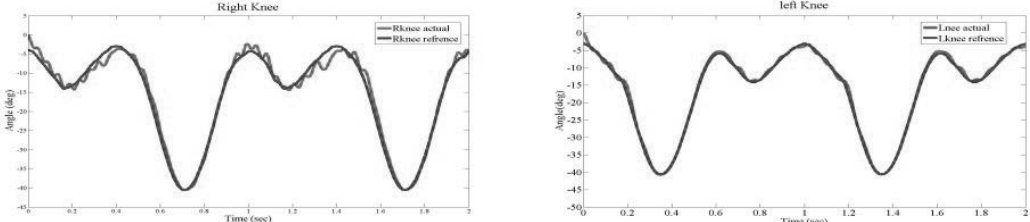

Figure 5. Hip joint trajectory tracking.

The torque of humanoid for hip and knee joints measured during the walking cycle is illustrated in Figure 6. It is noted that the torque for the hip joint was below $150 \mathrm{Nm}$ through the walking cycle and the torque for the knee joint was below $60 \mathrm{Nm}$ through the walking cycle. These conform to normal torque levels in practice.
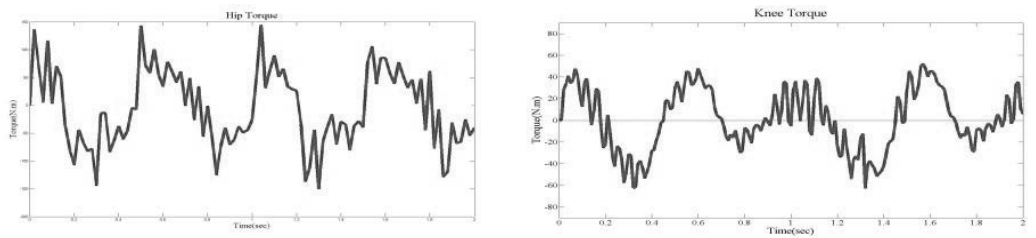

Figure 6. Torque profiles of humanoid during walking.

Figure 7 shows the torque profile of the hip and knee joints for the humanoid and exoskeleton. It is noted that the exoskeleton has provided the necessary augmentation to the humanoid joint torques during the walking cycle by supplying $25 \mathrm{Nm}$ to the knee joint and $60 \mathrm{Nm}$ to the hip joint. These are within of them are acceptable range as recommended by Low [18]; the maximum assistive torque to enhance the hip joint should be lower than 120 $\mathrm{Nm}$, and for knee joint should be lower than $60 \mathrm{Nm}$. The corresponding Visual Nastran captured image of the humanoid with exoskeleton during the walking cycle is shown in Figure 8. 

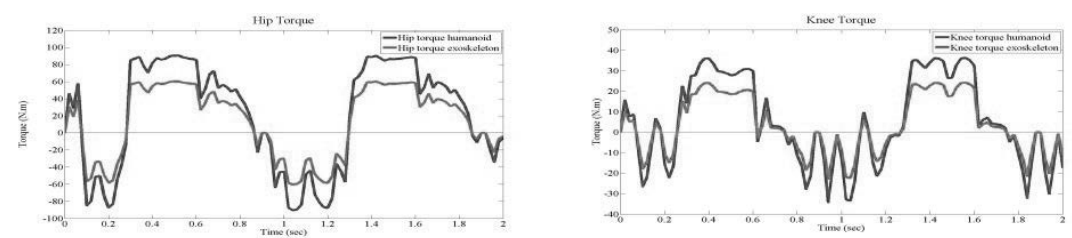

Figure 7. Torque profiles of humanoid and exoskeleton during walking.

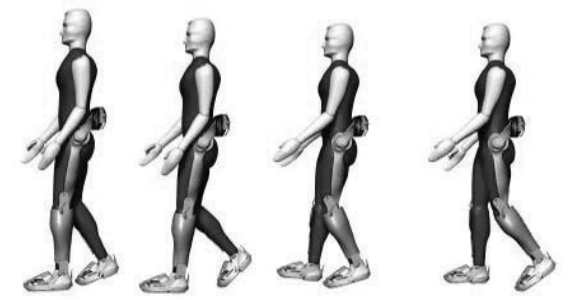

Figure 8. VN4D captured image during humanoid walking.

\section{Conclusions}

Investigations into development of assistive exoskeleton support for elderly mobility has been presented within simulation studies. Models of a humanoid and actuated lower limb exoskeleton device have been developed. Lower extremity exoskeleton has been developed to support the elderly mobility. A support torque of $40 \%$ of the total torque required for the walking cycle is provided by the exoskeleton. This has been achieved with a PD fuzzy logic control mechanism for the knee and hip joints. The results have shown that good trajectory tracking at both knee and hip joints has been achieved with torques below admissible limits for a human. Future work will investigate intelligent control paradigms with bio-inspired optimisation algorithms.

\section{References}

[1] U. Nations, World Population Ageing, 2013: UNITED NATIONS PUBN, 2014.

[2] S. M. Reza, N. Ahmad, I. A. Choudhury, and R. A. Ghazilla, "A fuzzy controller for lower limb exoskeletons during sit-to-stand and stand-to-sit movement using wearable sensors," Sensors (Basel), vol. 14, pp. 4342-63, 2014.

[3] H. Y. W. Xingsong, "kinematics analysis of lower extremity exoskeleton," Control and Decision, 2008.

[4] J. L. Pons, Wearable robots: biomechatronic exoskeletons vol. 338: Wiley Online Library, 2008. 
[5] Y. W. Hong, Y.-J. King, W.-H. Yeo, C.-H. Ting, Y.-D. Chuah, J.-V. Lee et al., "Lower Extremity Exoskeleton: Review and Challenges Surrounding the Technology and its Role in Rehabilitation of Lower Limbs," Australian Journal of Basic and Applied Sciences, vol. 7, pp. 520-524, 2013.

[6] G. Chen, C. K. Chan, Z. Guo, and H. Yu, "A review of lower extremity assistive robotic exoskeletons in rehabilitation therapy," Critical Reviews $^{\mathrm{TM}}$ in Biomedical Engineering, vol. 41, 2013.

[7] I. Díaz, J. J. Gil, and E. Sánchez, "Lower-Limb Robotic Rehabilitation: Literature Review and Challenges," Journal of Robotics, vol. 2011, pp. 1-11, 2011.

[8] J. E. Pratt, B. T. Krupp, C. J. Morse, and S. H. Collins, "The RoboKnee: an exoskeleton for enhancing strength and endurance during walking," in Robotics and Automation, 2004. Proceedings. ICRA'04. 2004 IEEE International Conference on, 2004, pp. 2430-2435.

[9] T. Yan, M. Cempini, C. M. Oddo, and N. Vitiello, "Review of assistive strategies in powered lower-limb orthoses and exoskeletons," Robotics and Autonomous Systems, vol. 64, pp. 120-136, 2015.

[10] B. Weinberg, J. Nikitczuk, A. Fisch, and C. Mavroidis, "Development of electro-rheological fluidic resistive actuators for haptic vehicular instrument controls," Smart materials and structures, vol. 14, p. 1107, 2005.

[11] H. Kawamoto and Y. Sankai, "Power assist system HAL-3 for gait disorder person," in Computers helping people with special needs, ed: Springer, 2002, pp. 196-203.

[12] T. Nakamura, K. Saito, W. Zhidong, and K. Kosuge, "Realizing modelbased wearable antigravity muscles support with dynamics terms," in Intelligent Robots and Systems, 2005. (IROS 2005). 2005 IEEE/RSJ International Conference on, 2005, pp. 2694-2699.

[13] T. Nakamura, K. Saito, and K. Kosuge, "Control of Wearable Walking Support System Based on Human-Model and GRF," in Robotics and Automation, 2005. ICRA 2005. Proceedings of the 2005 IEEE International Conference on, 2005, pp. 4394-4399.

[14] D. A. Winter, Biomechanics and motor control of human movement: John Wiley \& Sons, 2009.

[15] S. Wang, "Software review: Motion simulation with working model 2D and MSC visual Nastran 4D," Working Model 2D-Reviews and Awards, 2007.

[16] G. AL REZAGE, M. TOKHI, and S. K. ALI, "DESIGN AND CONTROL OF EXOSKELETON FOR ELDERLY MOBILITY," in ASSISTIVE 
ROBOTICS: Proceedings of the 18th International Conference on CLAWAR 2015, 2016, pp. 67-74.

[17] D. Pan, F. Gao, Y. Miao, and R. Cao, "Co-simulation research of a novel exoskeleton-human robot system on humanoid gaits with fuzzy-PID/PID algorithms," Advances in Engineering Software, vol. 79, pp. 36-46, 2015.

[18] K. Low, "Robot-assisted gait rehabilitation: From exoskeletons to gait systems," in Defense Science Research Conference and Expo (DSR), 2011, 2011, pp. 1-10. 